\title{
A new approach to maternal mortality: the role of HIV in pregnancy
}

\author{
This article was published in the following Dove Press journal: \\ International Journal of Women's Health \\ 28 May 2013 \\ Number of times this article has been viewed
}

\section{Sara E Gorman \\ Department of Epidemiology, Harvard University, Boston, MA, USA}

\begin{abstract}
As 2015 quickly approaches, we have been made increasingly aware of our progress toward Millennium Development Goals (MDGs). However, one MDG has been particularly recalcitrant to progress: MDG 5, namely, improving maternal health. Few countries are on track to achieve the first part of MDG 5's goals, reducing maternal mortality by $75 \%$. This article addresses the key priority issues of maternal health as part of sexual and reproductive health issues and maternal health and communicable diseases. It argues that only an integrative approach to the twin challenges of HIV and maternal mortality can help reduce devastatingly high rates of maternal deaths worldwide, especially in sub-Saharan Africa. The article reenvisions the MDGs not as separate, independent tasks, but as related, cohesive issues for which a holistic approach is needed. New causes of the relationship between HIV and maternal mortality are considered, and possible solutions are broached.
\end{abstract}

Keywords: HIV, maternal mortality, Millennium Development Goals, integrated approach to maternal health

\section{Introduction}

As 2015 quickly approaches, we have been made increasingly aware of our progress toward Millennium Development Goals (MDGs). Some remarkable progress has indeed been made. For example, the proportion of underweight children younger than age 5 in developing countries has declined from $28 \%$ to $17 \%$ between 1990 and $2011 .{ }^{1}$ Significant progress has also been made in reducing mortality among children under the age of 5 years. In 1990, 12 million such children under 5 years died, compared with 6.9 million children in $2011 .^{1}$ In $2011,2.5$ million people were newly infected with HIV, representing a $24 \%$ decrease from the 3.1 million people newly infected in 2001. ${ }^{1}$ However, one MDG has been particularly recalcitrant to progress: MDG 5, namely, improving maternal health. Improving maternal health involves not only reducing the maternal mortality ratio by $75 \%$ but also increasing the proportion of births attended by skilled health personnel and achieving universal access to reproductive health. Indicators for monitoring progress of universal access to reproductive health include contraceptive prevalence rate, adolescent birth rate, antenatal care coverage, and unmet need for family planning. Few countries are on track to achieve the first part of MDG 5's goals, reducing maternal morality by $75 \% .^{2}$ Sub-Saharan Africa is in the most dire position, with a regional maternal mortality rate of 640 maternal deaths out of 100,000 live births, and a decline rate of merely $0.1 \%$ per year. ${ }^{2}$ In the summer of 2012, the University of Cambridge hosted a conference on the topic New Approaches to Maternal Mortality - recognizing the crucial need to address the
Correspondence: Sara E Gorman Department of Epidemiology, Harvard University, 677 Huntington Avenue, Boston, MA 02115, USA Email saragorm@gmail.com 
problem of global maternal mortality rates and to address potential solutions. ${ }^{3}$

\section{HIV in pregnancy is contributing to high maternal mortality rates}

What is contributing to such high maternal mortality rates, and what can we do to help reduce them? In 2010, experts from WHO, UNICEF, United Nations Population Fund, and the World Bank came together to produce a report on global trends in maternal mortality. ${ }^{2}$ A relatively buried and subsequently unpublicized portion of the report revealed the key connection between maternal mortality and HIV infection. This key link has received little attention in both research and popular media, but the contribution of HIV infection to maternal morality is undoubtedly significant. The authors of the joint report estimated that in 2008 alone there were 42,000 deaths due to HIV/AIDS among pregnant women. ${ }^{2}$ Nine percent of all maternal deaths in sub-Saharan Africa were due to HIV in the period between 1990 and $2008 .^{2}$ In countries with high HIV prevalence, such as eastern and southern Africa, HIV has become the leading cause of death among women during pregnancy and the postpartum period. ${ }^{2}$

An even more recent study emphasized the clear and considerable contribution of HIV/AIDS to global maternal mortality rates. ${ }^{4}$ The authors of this meta-analysis found that a very high proportion of pregnancy-related deaths are attributable to HIV at the population level. They estimate that $5 \%$ of pregnancy-related deaths worldwide and $25 \%$ in sub-Saharan Africa are attributable to HIV. The authors note that the exact causes of maternal mortality among women with HIV/AIDS are unclear. Some have proposed that pregnancy might accelerate HIV progression or that the risk of obstetric complications may be increased in HIV-infected women. ${ }^{4}$ Yet the exact mechanism by which HIV contributes to maternal mortality is largely unknown, and further research is desperately needed.

\section{We need to understand better the link between HIV and maternal death}

Much more research is still needed on the biological basis of HIV-related maternal mortality. ${ }^{5}$ We know that the link exists, but we are still not entirely sure why it exists. ${ }^{6}$ Some progress has recently been made in understanding the connection between HIV and maternal death. One study that found a maternal mortality ratio ten times higher in HIV-infected pregnant women than in uninfected women in South Africa discovered that the most common causes of maternal death among HIV-infected pregnant women were nonpregnancy-related infections, such as AIDS, pneumonia, and tuberculosis, but that HIV-infected pregnant women were also at higher risk of dying from pregnancy-related sepsis and complications of abortion. ${ }^{7}$ These particular findings indicate a potential double burden for HIV-infected mothers: both improper treatment of and attention to their HIV infection as well as the possibility of HIV infection's direct contribution to pregnancy-related maternal deaths leave HIV-infected mothers much more vulnerable to postpartum complications than their uninfected counterparts.

In addition to understanding biological causes of HIV-related maternal mortality, greater comprehension of contextual factors that specifically reduce HIV-positive women's access to prenatal care is essential. Do HIVpositive pregnant women face stigma and discrimination in the health care system that prevents them from seeking the prenatal care they need? One prospective mixed-methods (combining qualitative and quantitative measures) study in Kenya found that women with higher perceptions of HIV-related stigma were less likely to deliver their babies in a health facility with a skilled attendant, often a crucial measure for reducing the occurrence of complications during childbirth. ${ }^{8}$ This avoidance could contribute to eventual obstetric complications and avoidable maternal deaths.

\section{Prenatal care and HIV treatment must be better integrated}

What would wider recognition of the crucial link between HIV and maternal mortality mean for interventions to reduce the maternal mortality ratio? For one thing, there should be an integrated approach to HIV and maternal health service delivery. Pregnant women with HIV worldwide should be given earlier access to antiretroviral treatment (ART) and access to family planning services. ${ }^{5}$ Although it is clear that better integration of HIV treatment and prenatal care is crucial, much work remains to be done to pinpoint exactly how this integration should be achieved. Cost-effectiveness of integrated services and health outcomes, such as HIV and sexually transmitted infection incidence and mortality, must continue to be investigated in order to inform the development of integrated programs and policies. ${ }^{9}$

Some strategies for integration of these services include lifelong ART for HIV-positive pregnant women, which can help prevent mother-to-child transmission as well as maternal mortality due to complications related to HIV in subsequent pregnancies and send a message to communities that ART 
should be continued for life. ${ }^{10}$ In countries with generalized HIV epidemics and antenatal HIV prevalence $>1 \%$, ART should be offered to eligible pregnant women in maternal health clinics, rather than in separate specialized HIV clinics, to reduce transportation costs, lower risk of attrition, and remove potentially stigmatizing obstacles. ${ }^{10}$ Preliminary investigations indicate that ART retention and treatment levels are higher among pregnant women attending maternal health services clinics that also provide HIV treatment services. ${ }^{10}$ Because many antenatal clinics lack the ability to perform $\mathrm{CD}^{+}$ T-lymphocyte counts, antenatal clinics in some countries, such as Malawi, have begun initiating all HIV-positive pregnant women on ART regardless of CD4 count. ${ }^{10}$ The results of this strategy have not yet been fully ascertained, but preliminary evidence suggests that this tactic might be effective.

Integrating ART and other HIV services into antenatal clinics will require training nurses, midwives, obstetricians, and gynecologists to provide HIV care and ART as well as HIV testing and counseling. Other health workers, including community health workers, might be needed in these integrated clinics to avoid overburdening nurses and midwives. This training may include developing standardized manuals for educating maternal health care providers about delivering HIV services. Clinics should be jointly owned by existing maternal and child health programs and HIV programs, integrating staff from both antenatal clinics and HIV clinics into one location. Ideally, electronic monitoring of ART should be available, and patient registers used in reproductive health and antenatal services should include information about HIV status and treatment. ${ }^{10}$ At the policy level, strategic planning and budgeting with relevant stakeholders for maternal health and HIV services will be necessary to help provide and sustain funds for integration of services, training of employees, and monitoring and evaluation. International aid agencies will need to be encouraged to provide funds specifically for integrated services, rather than focusing on one disease or health issue at a time. Some international donors are already taking notice of the need for funds directed specifically at integrated services of this kind. For example, at the 2010 Women Deliver conference, American businesswoman and philanthropist Melinda Gates pledged $\$ 1.5$ billion over the next 5 years to stimulate investment in integrated women's health programs, including programs that combine maternal health and HIV services. ${ }^{11}$ Communication and coordination across departments dealing with HIV and maternal health within local ministries of health must be strengthened, a process that is already under way in many countries. In order to avoid duplication and to maximize the use of available resources, national interagency coordination committees addressing the integration of these services should be formed, and continuous planning and management is essential. ${ }^{12}$

To aid pregnant women with disclosure of HIV status to their partners, clinics should offer couples testing and counseling at the first antenatal visit. Evidence has suggested that integrating couples counseling into antenatal care greatly increases pregnant women's uptake of efforts to prevent HIV transmission, including good ART retention. ${ }^{13}$ Since lack of partner support is associated with decreased use of ART, incorporating couples counseling into antenatal care for HIVpositive pregnant women might encourage usage of lifesaving therapies that may also help reduce maternal mortality rates. Couples counseling sessions may also be an ideal time to introduce the importance and effectiveness of ART in preventing transmission of HIV infection to the newborn child. Other possible interventions include integration of HIV services in general primary care or having HIV service providers visit antenatal clinics on specified days. However, fully integrated HIV and antenatal services at antenatal clinics probably have the greatest potential to control HIV infection among pregnant women, thereby reducing the chances of obstetric complications and maternal death. ${ }^{8}$

Greater attention to and greater provisions for HIV-specific services in young women of reproductive age could have a major impact on the global maternal mortality rate, especially in places like sub-Saharan Africa, where both HIV rates and the maternal mortality ratio are high. Moreover, progress on four main subgoals across two of the MDGs (5 and 6) will be enhanced by closer attention to treating, managing, and preventing HIV infection in pregnant women: reducing the maternal mortality ratio by $75 \%$; achieving universal access to reproductive health; halting and reversing the spread of HIV/AIDS; and achieving universal access to treatment for HIV/AIDS for all those who need it. Integrated maternal health and HIV services would help reduce rates of maternal death due to obstetric complications associated with HIV; would connect more women at risk with reproductive health services, including prevention and treatment of sexually transmitted infections other than HIV; would control HIV infection among a vulnerable population of sexually active women and prevent transmission to HIV-negative partners and mother-to-child transmission; and would help connect a large population of HIV-positive women with treatment.

In broader terms, it may be wise to take a more integrated approach to the MDGs, recognizing that they may not be as isolated from each other as they might seem. MDG 6, 
combating HIV/AIDS, malaria, and other diseases, should be integrated with MDG 5, improving maternal health and reducing maternal mortality. Part of the goals of MDG 6 would directly influence efforts to reduce maternal mortality, including reducing HIV prevalence in people aged 15-24 years and increasing the proportion of the population with advanced HIV infection with access to antiretroviral drugs. The MDGs cannot be achieved in isolation from each other; in fact, MDGs 3 (eliminate sex disparities in education), 4 (reduce the under-five mortality rate by two-thirds), 5, and 6 are all connected in vital ways. Promoting equality and empowering women through better education and higher wages is directly related to women's ability to seek and obtain better antenatal care, to prevent and treat HIV infection that contributes to maternal mortality, and to reduce the incidence of obstetric complications that contribute to both maternal and child mortality. To be sure, goals of reducing incidence of HIV and reducing maternal mortality rates are inextricably linked.

\section{Disclosure}

The author declares no conflicts of interest in this work.

\section{References}

1. World Health Organization. Millennium Development Goals (MDGs). 2012. Available from: http://www.who.int/mediacentre/factsheets/fs290/ en/index.html. Accessed March 20, 2013.

2. World Health Organization. Trends in Maternal Mortality: 1990 to 2008. Geneva: WHO; 2010. Available from: http://whqlibdoc.who.int/publications/2010/9789241500265_eng.pdf. Accessed April 24, 2013.
3. Centre for Research in the Arts, Social Sciences and Humanities, University of Cambridge. New approaches to maternal mortality in Africa. 2012. Available from: http://www.crassh.cam.ac.uk/ events/1977. Accessed March 20, 2013.

4. Calvert C, Ronsmans C. The contribution of HIV to pregnancyrelated mortality: a systematic review and meta-analysis. AIDS. Epub February 25, 2013.

5. Abdool-Karim Q, AbouZhar C, Dehne K, et al. HIV and maternal mortality: turning the tide. Lancet. 2010;375(9730):1948-1949.

6. Landes M, van Lettow M, Bedell R, et al. Mortality and health outcomes in HIV-infected and HIV-uninfected mothers at 18-20 months postpartum in Zomba District, Malawi. PLoS One. 2012;7(9):e44396.

7. Moran NF, Moodley J. The effect of HIV infection on maternal health and mortality. Int J Gynaecol Obstet. 2012;119 Suppl 1:S26-S29.

8. Turan JM, Hatcher AH, Medema-Wijnveen J, et al. The role of HIVrelated stigma in utilization of childbirth services in rural Kenya: a prospective mixed-methods study. PLoS Med. 2012;9(8):e1001295.

9. Lindegren ML, Kennedy CE, Bain-Brickley D, et al. Integration of HIV/ AIDS services with maternal, neonatal and child health, nutrition, and family planning services. Cochrane Database Syst Rev. 2012;12(9): CD010119.

10. Suthar AM, Hoos D, Beqiri A, et al. Integrating antiretroviral therapy into antenatal care and maternal and child health settings: a systematic review and meta-analysis. Bull World Health Organ. 2013;91(1): $46-56$.

11. PLoS Medicine Editors. HIV in maternal and child health: concurrent crises demand cooperation. PLoS Med. 2010;7(7):e1000311.

12. World Health Organization. Technical Consultation on the Integration of HIV Interventions into Maternal, Newborn and Child Health Services. Geneva: WHO; 2008. Available from: http://apps.who.int/ iris/bitstream/10665/69767/1/WHO_MPS_08.05_eng.pdf. Accessed April 23, 2013.

13. Farquhar C, Kiarie JN, Richardson BA, et al. Antenatal couple counseling increases uptake of interventions to prevent HIV-1 transmission. J Acquir Immune Defic Syndr. 2004;37(5):1620-1626.
International Journal of Women's Health

\section{Publish your work in this journal}

The International Journal of Women's Health is an international, peerreviewed open-access journal publishing original research, reports, editorials, reviews and commentaries on all aspects of women's healthcare including gynecology, obstetrics, and breast cancer. The manuscript management system is completely online and includes

\section{Dovepress}

a very quick and fair peer-review system, which is all easy to use. Visit http://www.dovepress.com/testimonials.php to read real quotes from published authors. 